\title{
Restricted versions of the Tukey-Teichmüller Theorem that are equivalent to the Boolean Prime Ideal Theorem
}

\author{
R.E. Hodel
}

Dedicated to W.W. Comfort on the occasion of his seventieth birthday.

\begin{abstract}
We formulate a restricted version of the Tukey-Teichmüller Theorem that we denote by (rTT). We then prove that (rTT) and (BPI) are equivalent in $\mathrm{ZF}$ and that (rTT) applies rather naturally to several equivalent forms of (BPI): Alexander Subbase Theorem, Stone Representation Theorem, Model Existence and Compactness Theorems for propositional and first-order logic. We also give two variations of $(\mathrm{rTT})$ that we denote by $(\mathrm{rTT})^{+}$and $(\mathrm{rTT})^{++}$; each is equivalent to (rTT) in $\mathrm{ZF}$. The variation $(\mathrm{rTT})^{++}$applies rather naturally to various Selection Lemmas due to Cowen, Engeler, and Rado.
\end{abstract}

\section{Introduction}

Let ZF denote Zermelo-Fraenkel set theory. It is well known that the axiom of choice $(\mathrm{AC})$ is independent of $\mathrm{ZF}$. When we add $\mathrm{AC}$ to $\mathrm{ZF}$ we obtain the most widely used axiom system for classical mathematics, namely

$$
\mathrm{ZFC}=\mathrm{ZF}+\mathrm{AC} .
$$

It is also well known that there are many theorems of ZFC that are in fact equivalent forms of AC. For example, we have the following short list (see [12], [13], and [19] for much longer lists):

Theorem 1.1 (ZF) The following are equivalent:

(1) the axiom of choice $(A C)$;

(2) the well-ordering principle;

(3) Zorn's Lemma;

(4) Tychonoff Product Theorem;

(5) Tukey-Teichmüller Theorem (TT). 
In this paper we are especially interested in (TT). Recall that a collection $\mathcal{A}$ of subsets of a set $X$ has finite character if for every $A \subseteq X, A \in \mathcal{A}$ if and only if every finite subset of $A$ is in $\mathcal{A}$. Note that $\varnothing \in \mathcal{A}$ (assuming that $\mathcal{A}$ is non-empty). There are two versions of the Tukey-Teichmüller Theorem; however, in ZF they are equivalent to each other and also to AC.

TT (weak form) Let $X$ be a set and let $\mathcal{A}$ be a non-empty collection of subsets of $X$ with finite character. Then $\mathcal{A}$ has a maximal element (with respect to $\subseteq$ ).

TT (strong form) Let $X$ be a set and let $\mathcal{A}$ be a non-empty collection of subsets of $X$ with finite character. Then for each $A \in \mathcal{A}$, there exists $B \in \mathcal{A}$ such that $A \subseteq B$ and $B$ is maximal.

The Tukey-Teichmüller Theorem seems tailor-made for certain applications. For example, it can be used to prove that every vector space has a basis by simply noting that the property of being linearly independent has finite character. Another nice application is a proof of the Alexander Subbase Theorem (see [14] or [7]).

The axiom of choice and its equivalent forms are non-constructive principles; they assert the existence of a set without giving instructions on how to construct the set. There are other such non-constructive principles that are perhaps not so well known as those given above. Here is a short list (see [12], form 14, for a much longer list; additional references are [2], [3], [13], [18], and [20]).

Theorem 1.2 (ZF) The following are equivalent:

(1) every ideal in a Boolean algebra extends to a prime ideal (BPI);

(2) ever filter on a set extends to an ultrafilter (UT);

(3) Alexander Subbase Theorem;

(4) Stone Representation Theorem;

(5) Compactness Theorem for propositional logic;

(6) Model Existence Theorem for propositional logic.

The logical status of these ideas is not trivial. The easy part is:

$$
\mathrm{ZF}+\mathrm{AC} \Rightarrow \mathrm{ZF}+\mathrm{BPI} \Rightarrow \mathrm{ZF}
$$

The hard part states:

- AC cannot be proved from ZF + BPI (Halpern and Lévy [10]);

- BPI cannot be proved from ZF (S. Feferman [8]).

In section 2 we formulate a version of the Tukey-Teichmüller Theorem (TT) that is equivalent to (BPI) rather than to (AC). We then use this new 
non-constructive axiom, denoted (rTT) (for restricted Tukey-Teichmüller), to give direct proofs of the Alexander Subbase Theorem and the Stone Representation Theorem. In section 3 we show directly that (rTT) and (BPI) are equivalent in ZF. Finally, in section 4 we use (rTT) to derive various theorems of logic that are known to be equivalent to (BPI).

\section{Statement of (rTT)}

To motivate the statement of (rTT), let us use (TT) to prove the Boolean prime ideal theorem. Here are the required definitions. Let $\left\langle B, \vee, \wedge,,^{\prime}, 0,1\right\rangle$ be a Boolean algebra and let $J$ be a subset of $B$.

- $J$ is an ideal of $B$ if $0 \in J, 1 \notin J$, and the following hold for all $a, b \in B$ :

(a) if $a, b \in J$, then $a \vee b \in J$; (b) if $a \in J$ and $b \in B$, then $a \wedge b \in J$.

- $J$ is a prime ideal if $J$ is an ideal and satisfies: if $a \wedge b \in J$, then $a \in J$ or $b \in J$.

- $J$ has the finite join property if $a_{1} \vee \cdots \vee a_{n} \neq 1$ for all $a_{1}, \cdots, a_{n} \in J$.

Note the following: If $J$ has the finite join property, and for all $a \in B$, either $a \in J$ or $a^{\prime} \in J$, then $J$ is a prime ideal. The following lemma plays a key role in our formulation of (rTT).

Lemma 2.1 (extension property) If $J \subseteq B$ has the finite join property and $x \in B$, then $J \cup\{x\}$ or $J \cup\left\{x^{\prime}\right\}$ has the finite join property.

Proof. Suppose that neither set has the finite join property. Then there exist $a_{1}, \cdots, a_{n}, b_{1}, \cdots, b_{k} \in J$ such that

$$
a_{1} \vee \cdots \vee a_{n} \vee x=1 \text { and } b_{1} \vee \cdots \vee b_{k} \vee x^{\prime}=1
$$

It follows that $z \vee x=1$ and $z \vee x^{\prime}=1$, where

$$
z=a_{1} \vee \cdots \vee a_{n} \vee b_{1} \vee \cdots \vee b_{k}
$$

But from $z \vee x=1$ and $z \vee x^{\prime}=1$ we obtain $z=1$, a contradiction to the assumption that $J$ has the finite join property.

Theorem 2.2 (Boolean Prime Ideal) Every ideal of a Boolean algebra is contained in a prime ideal.

Proof (using (TT)). Let $I$ be an ideal of a Boolean algebra $B$. Let

$$
\mathcal{A}=\{J: J \subseteq B \text { and } J \text { has the finite join property }\} .
$$


The collection $\mathcal{A}$ has finite character and $I \in \mathcal{A}$; by (TT) there exists $J \in \mathcal{A}$ such that $I \subseteq J$ and $J$ is maximal (with respect to $\subseteq$ ). Let $x \in B$; by the extension property (Lemma 2.1), $J \cup\{x\}$ or $J \cup\left\{x^{\prime}\right\}$ has the finite join property. By the maximality of $J, x \in J$ or $x^{\prime} \in J$. It follows that $J$ is the required prime ideal.

We now state the hypotheses of (rTT) and two conclusions (I) and (II).

(rTT) Let $X$ be a set, let $\mathcal{A}$ be a non-empty collection of subsets of $X$, and let' be a 1-ary operation on $X$. Assume that

(1) $\mathcal{A}$ has finite character;

(2) $\mathcal{A}$ has the extension property with respect to ' (for all $A \in \mathcal{A}$ and all $x \in X, A \cup\{x\} \in \mathcal{A}$ or $\left.A \cup\left\{x^{\prime}\right\} \in \mathcal{A}\right)$.

(I) There exists $B \in \mathcal{A}$ such that for all $x \in X, x \in B$ or $x^{\prime} \in B$.

(II) For all $A \in \mathcal{A}$, there exists $B \in \mathcal{A}$ such that $A \subseteq B$ and for all $x \in X$, $x \in B$ or $x^{\prime} \in B$.

We have stated two versions of (rTT), the weak version (conclusion (I)) and the strong version (conclusion (II)). Actually, the two versions are equivalent in $\mathrm{ZF}$.

To derive the strong version from the weak, let $\mathcal{A}$ be a non- empty collection of subsets of $X$ such that $\mathcal{A}$ has finite character and the extension property with respect to a 1 -ary operation ' on $X$. Given $A \in \mathcal{A}$, let

$$
\mathcal{B}=\{B: B \subseteq X \text { and } A \cup B \in \mathcal{A}\}
$$

The collection $\mathcal{B}$ satisfies all of the hypotheses of (rTT). It follows from (I) that there exists $B \in \mathcal{B}$ such that for all $x \in X$, either $x \in B$ or $x^{\prime} \in B$. It is easy to check that $A \cup B$ is the required element of $\mathcal{A}$.

Note that $(\mathrm{TT}) \Rightarrow(\mathrm{rTT})$. For, suppose that we have $B \in \mathcal{A}$ such that $B$ is maximal (with respect to $\subseteq$ ). From the extension property it follows that for all $x \in X$, either $x \in B$ or $x^{\prime} \in B$. We emphasize that

(rTT) is an equivalent form of (BPI) that follows easily from a well-known equivalent form of the axiom of choice.

We now show that the Alexander Subbase Theorem and the Stone Representation Theorem follow rather naturally from (rTT). In all applications of (rTT), the theme is:

finite character + extension property (with respect to a 1-ary operation). 
In this paper the 1-ary operation will always be the complement operation in a Boolean algebra or the negation operation in a formal language.

$(\mathbf{r T T}) \Rightarrow$ Alexander Subbase Theorem We will show that the proof in [14] goes through with (TT) replaced with (rTT). The following terminology is used. A collection $\mathcal{W}$ of subsets of a set $X$ is finitely inadequate if no finite subcollection of $\mathcal{W}$ covers $X$. (This is just the finite join property for the Boolean algebra $P(X)$.) The property of being finitely inadequate satisfies the following conditions:

(1) finite character $[\mathcal{W}$ is finitely inadequate if and only if every finite subset of $\mathcal{W}$ is finitely inadequate];

(2) extension property [if $\mathcal{W}$ is finitely inadequate and $E \subseteq X$, then either $\mathcal{W} \cup\{E\}$ or $\mathcal{W} \cup\left\{E^{c}\right\}$ is finitely inadequate].

Theorem 2.3 (Alexander Subbase) Let $X$ be a topological space and let $\mathcal{S}$ be a subbase for $X$ such that for every subcollection $\mathcal{S}_{0}$ of $\mathcal{S}$ that covers $X$, there is a finite subcollection of $\mathcal{S}_{0}$ that covers $X$. Then $X$ is compact.

Proof. Let $\mathcal{W}$ be an open cover of $X$. Suppose, by way of contradiction, that no finite subcollection of $\mathcal{W}$ covers $X$; in other words, $\mathcal{W}$ is finitely inadequate. Let

$$
\begin{gathered}
\mathcal{B}=\left\{B: B \subseteq W \text { for some } W \in \mathcal{W} \text { and } B=S_{1} \cap \cdots \cap S_{n}\right. \text {, where } \\
\left.\qquad S_{1}, \cdots, S_{n} \in \mathcal{S}\right\}
\end{gathered}
$$

The collection $\mathcal{B}$ covers $X$ and is finitely inadequate (since $\mathcal{W}$ has these two properties). By (rTT), there exists $\mathcal{C} \subseteq P(X)$ such that

(1) $\mathcal{B} \subseteq \mathcal{C}$;

$(2) \mathcal{C}$ is finitely inadequate;

(3) for all $E \subseteq X, E \in \mathcal{C}$ or $E^{c} \in \mathcal{C}$.

For each $B \in \mathcal{B}$, there exists $S_{B} \in \mathcal{C} \cap \mathcal{S}$ such that $B \subseteq S_{B}$. [Details: $B=S_{1} \cap \cdots \cap S_{n}$, where $S_{1}, \cdots, S_{n} \in \mathcal{S}$; by (1), (2) and (3), $S_{k} \in \mathcal{C}$ for some $k \leq n$. The axiom of choice for finite sets (ACF) is used here; more on this later.] We now have a subcollection $\mathcal{S}_{0}=\left\{S_{B}: B \in \mathcal{B}\right\}$ of $\mathcal{S}$ that covers $X$. By hypothesis, there is a finite subcollection $\mathcal{F}$ of $\mathcal{S}_{0}$ that covers $X$. But $\mathcal{F} \subseteq \mathcal{C}$ and we have contradicted the fact that $\mathcal{C}$ is finitely inadequate.

$(\mathbf{r T T}) \Rightarrow$ Stone Representation Theorem Next we use (rTT) to prove the Stone Representation Theorem, the most important structural theorem about Boolean algebras. It asserts that every Boolean algebra is isomorphic to a field of sets. We will approach the construction from the following point 
of view: Given a structure $\left\langle B, \wedge,{ }^{\prime}, 0\right\rangle$, where $B$ has more than one element, what axioms are required to show that $B$ is isomorphic to a field of sets? These axioms will automatically make $B$ a Boolean algebra, where $a \vee b$ is defined as $\left(a^{\prime} \wedge b^{\prime}\right)^{\prime}$ and 1 is defined as $0^{\prime}$. To begin with, we will want the following:

$(\mathrm{B} 1) \wedge$ is commutative, associative, and idempotent;

(B2) for all $x \in B, x \wedge x^{\prime}=0$.

Moreover, in the process of proving the Stone Representation Theorem, we will see that two additional properties are required, namely

(B3) if $a \wedge x=0$ and $a \wedge x^{\prime}=0$, then $a=0$;

(B4) if $a \wedge b^{\prime}=0$ and $a^{\prime} \wedge b=0$, then $a=b$.

Two definitions are required; the first is the dual of the finite join property that was defined in connection with (BPI). Let $U \subseteq B$.

- $U$ has the finite meet property if $a_{1} \wedge \cdots \wedge a_{n} \neq 0$ for all $a_{1}, \cdots, a_{n} \in U$;

- $U$ is an ultrafilter if $U$ has the finite meet property and for all $x \in B, x \in U$ or $x^{\prime} \in U$.

The finite meet property has finite character, and axiom (B3) proves that it also has the extension property with respect to the 1-ary operation ' (see the proof of Lemma 2.1). Hence (rTT) applies and we can assert: every subset of $B$ with the finite meet property is contained in an ultrafilter.

Theorem 2.4 (Stone Representation) Let $\left\langle B, \wedge,,^{\prime}, 0\right\rangle$ be a structure that satisfies $(B 1)-(B 4)$. Then $B$ is isomorphic to a field of sets, and hence $B$ is a Boolean algebra, where $a \vee b=\left(a^{\prime} \wedge b^{\prime}\right)^{\prime}$ and $1=0^{\prime}$.

Proof. Let $U L T(B)=\{U: U$ is an ultrafilter on $B\}$, and define $\Phi: B \rightarrow$ $P(U L T(B))$ by

$$
\Phi(a)=\{U: U \text { is an ultrafilter on } B \text { and } a \in U\} .
$$

Then

(1) $\Phi(a \wedge b)=\Phi(a) \cap \Phi(b)$;

(2) $\Phi\left(a^{\prime}\right)=U L T(B)-\Phi(a)$.

The proof is complete if we can prove that $\Phi$ is one-to-one. Let $a \neq b$. By (B4), $a \wedge b^{\prime} \neq 0$ or $a^{\prime} \wedge b \neq 0$. Suppose that $a \wedge b^{\prime} \neq 0$. Then $\left\{a, b^{\prime}\right\}$ has the finite meet property and so there is an ultrafilter $U$ such that $\left\{a, b^{\prime}\right\} \subseteq U$. We now have $a \in U$ and $b \notin U$; hence $\Phi(a) \neq \Phi(b)$ as required.

We have given a non-constructive proof that (B1)-(B4) is a suitable set of axioms for a Boolean algebra. Frink [9] has used the same method (with Zorn's Lemma) to give a similar list of axioms. In a subsequent paper we will give a constructive proof that these axioms suffice and also give applications of this axiom set. 


\section{3 (rTT) and (BPI) are equivalent in $\mathrm{ZF}$}

It is convenient to work with the dual of the (BPI). The special case that we need is called the Ultrafilter Theorem (UT) and can be stated in terms of the following definitions. Let $X$ be a non-empty set and let $\mathcal{U}$ be a non-empty collection of subsets of $X$.

- $\mathcal{U}$ has the finite intersection property (FIP) if $F_{1} \cap \cdots \cap F_{n} \neq \varnothing$ for every finite subcollection $\left\{F_{1}, \cdots, F_{n}\right\}$ of $\mathcal{U}$;

- $\mathcal{U}$ is an ultrafilter if $\mathcal{U}$ has the FIP and for all $U \subseteq X, U \in \mathcal{U}$ or $U^{c} \in \mathcal{U}$.

An ultrafilter $\mathcal{U}$ on $X$ has the following property: if $\left\{Y_{1}, \cdots, Y_{n}\right\}$ is a pairwise disjoint collection of subsets of $X$ such that $X=Y_{1} \cup \cdots \cup Y_{n}$, then there is a unique $k \leq n$ such that $Y_{k} \in \mathcal{U}$.

Theorem 3.1 (Ultrafilter) Let $X$ be a non-empty set and let $\mathcal{F}$ be a nonempty collection of subsets of $X$ with the finite intersection property. Then there is an ultrafilter $\mathcal{U}$ on $X$ such that $\mathcal{F} \subseteq \mathcal{U}$.

Before proving the equivalence of (rTT) and (UT), we formulate two variations of $(\mathrm{rTT})$ that we denote by $(\mathrm{rTT})^{+}$and $(\mathrm{rTT})^{++}$. The basic idea in each case is to replace the 1 -ary operation ' on $X$ with a collection $\mathcal{Z}=$ $\left\{Z_{t}: t \in T\right\}$, where each $Z_{t}$ is a finite subset of $X$.

$(\mathbf{r T T})^{+}$Let $X$ be a set, let $\mathcal{A}$ be a non-empty collection of subsets of $X$ that has finite character, and let $\mathcal{Z}=\left\{Z_{t}: t \in T\right\}$ be a collection of finite subsets of $X$. Assume that

$(+)$ for all $A \in \mathcal{A}$ and all $t \in T$, there exists $z \in Z_{t}$ such that $A \cup\{z\} \in \mathcal{A}$.

Then there exists $B \in \mathcal{A}$ such that $B \cap Z_{t} \neq \varnothing$ for all $t \in T$.

Note that (rTT) follows immediately from (rTT) $)^{+}$by taking $\mathcal{Z}=\left\{\left\{x, x^{\prime}\right\}\right.$ : $x \in X\}$. The following form of the axiom of choice for finite sets is an easy consequence of $(\mathrm{rTT})^{+}$: if $\left\{Z_{t}: t \in T\right\}$ is a pairwise disjoint collection of finite and non-empty sets, then there is a set $B$ that intersects each $Z_{t}$ in a singleton.

The variation $(\mathrm{rTT})^{+}$, like (rTT), has a weak version and a strong version that are actually equivalent in $\mathrm{ZF}$. The conclusion of the strong version states: For all $A \in \mathcal{A}$, there exists $B \in \mathcal{A}$ such that $A \subseteq B$ and for all for all $t \in T$, $B \cap Z_{t} \neq \varnothing$.

We now state a generalization of $(\mathrm{rTT})^{+}$.

$(\text { rTT })^{++}$Let $X$ be a set, let $\mathcal{A}$ be a non-empty collection of subsets of $X$ that has finite character, and let $\mathcal{Z}=\left\{Z_{t}: t \in T\right\}$ be a collection of finite subsets of $X$. Assume that 
$(++)$ for each finite subset $\left\{t_{1}, \cdots, t_{n}\right\}$ of $T$, there is a set $\left\{z_{1}, \cdots, z_{n}\right\} \in \mathcal{A}$ such that $z_{k} \in Z_{t_{k}}$ for $1 \leq k \leq n$.

Then there exists $B \in \mathcal{A}$ such that $B \cap Z_{t} \neq \varnothing$ for all $t \in T$.

Note that condition $(++)$ follows from $(+)$. For, let $\left\{t_{1}, \cdots, t_{n}\right\}$ be a finite subset of $T$. Since the collection $\mathcal{A}$ is non-empty and has finite character, $\varnothing \in \mathcal{A}$. Apply $(+)$ to $\varnothing$ and $t_{1}$ : there exists $z_{1} \in Z_{t_{1}}$ such that $\varnothing \cup\left\{z_{1}\right\} \in$ $\mathcal{A}$; in other words, $\left\{z_{1}\right\} \in \mathcal{A}$. Continue this a total of $n$ times to obtain $\left\{z_{1}, \cdots, z_{n}\right\} \in \mathcal{A}$ with $z_{k} \in Z_{t_{k}}$ for $1 \leq k \leq n$ as required.

Later we will give applications of $(\mathrm{rTT})^{++}$. But first we prove the following theorem.

Theorem 3.2 (ZF) The following are equivalent:

(1) $(r T T)^{++}$

(2) $(r T T)^{+}$

(3) the restricted Tukey-Teichmüller Theorem (rTT);

(4) Ultrafilter Theorem (UT).

Proof. We have already seen that $(\mathrm{rTT})^{++} \Rightarrow(\mathrm{rTT})^{+}$and that $(\mathrm{rTT})^{+} \Rightarrow$ $(\mathrm{rTT})$. Moreover, $(\mathrm{rTT}) \Rightarrow(\mathrm{UT})$ is essentially Theorem 2.2. It remains to prove that $(\mathrm{UT}) \Rightarrow(\mathrm{rTT})^{++}$. In this proof we will use the well known result that the Ultrafilter Theorem implies the axiom of choice for finite sets (ACF); at the end of this section we will sketch a proof of this implication. Let $\mathcal{A} \subseteq P(X)$ be non-empty and have finite character and let $\mathcal{Z}=\left\{Z_{t}: t \in T\right\}$ be a collection of finite subsets of $X$ that satisfies the condition $(++)$. We are required to show that there exists $B \in \mathcal{A}$ such that $B \cap Z_{t} \neq \varnothing$ for all $t \in T$. Let

$$
Y=\prod_{t \in T} Z_{t}
$$

and let $g \in Y$ (by ACF). For each $F \in[T]^{<\omega}$ (i.e., for each finite subset $F$ of $T$ ), let

$$
H_{F}=\{f \in Y:\{f(t): t \in F\} \in \mathcal{A}\}
$$

and let

$$
\mathcal{H}=\left\{H_{F}: F \in[T]^{<\omega}\right\} .
$$

The collection $\mathcal{H}$ satisfies the following two properties:

(a) $H_{F} \neq \varnothing$ for all $F \in[T]^{<\omega}$;

(b) $H_{F \cup G} \subseteq H_{F} \cap H_{G}$ for all $F, G \in[T]^{<\omega}$. 
Part (a) follows immediately from $(++)$ and the existence of $g \in Y$; part (b) uses the fact that $\mathcal{A}$ has finite character. It follows from (a) and (b) that the collection $\mathcal{H}$ has the finite intersection property. By (UT), there is an ultrafilter $\mathcal{U}$ on $Y$ such that $H_{F} \in \mathcal{U}$ for all $F \in[T]^{<\omega}$.

Claim For each $t \in T$, there is a unique $z_{t} \in Z_{t}$ such that $Y_{t} \in \mathcal{U}$, where $Y_{t}=\left\{f: f \in Y\right.$ and $\left.f(t)=z_{t}\right\}$.

To see this, let $t \in T$, let $Z_{t}=\left\{z_{1}, \cdots, z_{n}\right\}$, and for $1 \leq k \leq n$ let

$$
Y_{k}=\left\{f: f \in Y \text { and } f(t)=z_{k}\right\} \text {. }
$$

The collection $\left\{Y_{1}, \cdots, Y_{n}\right\}$ is pairwise disjoint and $Y_{1} \cup \cdots \cup Y_{n}=Y$; since $\mathcal{U}$ is an ultrafilter on $Y$, there is a unique integer $k(t), 1 \leq k(t) \leq n$, such that $Y_{k(t)} \in \mathcal{U}$. The required unique element of $Z_{t}$ is $z_{k(t)}$.

Now let

$$
B=\left\{z_{t}: t \in T\right\} .
$$

Clearly $B \cap Z_{t} \neq \varnothing$ for all $t \in T$. To see that $B \in \mathcal{A}$, it suffices by the finite character of $\mathcal{A}$ to show that $\left\{z_{t_{1}}, \cdots, z_{t_{n}}\right\} \in \mathcal{A}$. Let $F=\left\{t_{1}, \cdots, t_{n}\right\}$, and for $1 \leq k \leq n$ let

$$
Y_{t_{k}}=\left\{f: f \in Y \text { and } f\left(t_{k}\right)=z_{t_{k}}\right\}
$$

By the Claim, $Y_{t_{k}} \in \mathcal{U}$ for $1 \leq k \leq n$. Now $H_{F}$ is also in $\mathcal{U}$, and so there is some $f \in Y$ such that

$$
f \in H_{F} \cap Y_{t_{1}} \cap \cdots \cap Y_{t_{n}}
$$

Since $f \in H_{F},\left\{f\left(t_{1}\right), \cdots, f\left(t_{n}\right)\right\} \in \mathcal{A}$. But $f\left(t_{k}\right)=z_{t_{k}}$ for $1 \leq k \leq n$ and so $\left\{z_{t_{1}}, \cdots, z_{t_{n}}\right\} \in \mathcal{A}$ as required.

We now show that various Selection Lemmas due to Cowen, Engeler, and Rado follow rather naturally from $\left(\mathrm{rTT}^{++}\right.$. The first result is listed as $14 \mathrm{X}$, p. 22 of [12] and can be stated as follows.

Theorem 3.3 (Cowen-Engeler) Let $T$ and $X$ be non-empty sets and let $\mathcal{E}$ be a collection of functions from subsets of $T$ into $X$ such that

(1) $\mathcal{E}$ has finite character (that is, a function $\phi$ from a subset of $T$ into $X$ is in $\mathcal{E}$ if and only if for every finite $F \subseteq$ dom $\phi, \phi \mid F$ is in $\mathcal{E})$;

(2) for all $F \in[T]^{<\omega}$, there is a function $\phi \in \mathcal{E}$ whose domain is $F$;

(3) for all $t \in T$, the set $X_{t}=\{\phi(t): \phi \in \mathcal{E}$ and $t \in$ dom $\phi\}$ is finite.

Then $T$ is the domain of some $\phi \in \mathcal{E}$. 
To emphasize the close connection between $(\mathrm{rTT})^{++}$and the Cowen-Engeler Theorem, we will give a direct proof that the two are equivalent in $\mathrm{ZF}$.

Proof that $(\mathbf{r T T})^{++} \Rightarrow$ Cowen-Engeler. Let $\mathcal{E}$ be a collection of functions from subsets of $T$ into $X$ that satisfy (1)-(3) of the Cowen-Engeler Thorem. For each $t \in T$ let

$$
Z_{t}=\left\{\langle t, x\rangle: x \in X_{t}\right\}
$$

By $(3), Z_{t}$ is finite. To apply $(\mathrm{rTT})^{++}$, it suffices to check $(++)$. Let $F=$ $\left\{t_{1}, \cdots, t_{n}\right\} \subseteq T$. By $(2)$, there is a function $\phi \in \mathcal{E}$ whose domain is $F$. We now have

$$
\phi=\left\{\left\langle t_{1}, \phi\left(t_{1}\right)\right\rangle, \cdots,\left\langle t_{n}, \phi\left(t_{n}\right)\right\rangle\right\} \in \mathcal{E} \text { and }\left\langle t_{k}, \phi\left(t_{k}\right)\right\rangle \in Z_{t_{k}} \text { for } 1 \leq k \leq n .
$$

By $(\mathrm{rTT})^{++}$, there exists $\phi \in \mathcal{E}$ such that $\phi \cap Z_{t} \neq \varnothing$ for all $t \in T$; in other words, the domain of $\phi$ is $T$ as required.

Proof that Cowen-Engeler $\Rightarrow(\mathbf{r T T})^{++}$. Let $\mathcal{A}$ be a non-empty collection of subsets of $X$ with finite character and let $\left\{Z_{t}: t \in T\right\}$ be a collection of finite subsets of $X$ such that the condition $(++)$ holds. We are required to find $B \in \mathcal{A}$ such that $B \cap Z_{t} \neq \varnothing$ for all $t \in T$. Let

$$
\begin{gathered}
\mathcal{E}=\left\{\phi: \phi \text { is a function with } \operatorname{dom} \phi \subseteq T, \phi(t) \in Z_{t} \text { for all } t \in \operatorname{dom} \phi,\right. \text { and } \\
\text { for every finite } F \subseteq \operatorname{dom} \phi,\{\phi(t): t \in F\} \in \mathcal{A}\} .
\end{gathered}
$$

The collection $\mathcal{E}$ has the following properties:

(1) finite character;

(2) for all $F \in[T]^{<\omega}$, there is $\phi \in \mathcal{E}$ with dom $\phi=F$ (follows from $(++)$ and the finite character of $\mathcal{A}$ );

(3) for all $t \in T,\{\phi(t): \phi \in \mathcal{E}$ and $t \in \operatorname{dom} \phi\} \subseteq Z_{t}$.

By the Cowen-Engeler Theorem, there exists $\phi \in \mathcal{E}$ such that dom $\phi=T$. Let

$$
B=\{\phi(t): t \in T\} .
$$

Clearly $B \cap Z_{t} \neq \varnothing$ for all $t \in T$. To show that $B \in \mathcal{A}$, it suffices to show that each finite subset of $B$ is in $\mathcal{A}$. Let $\left\{t_{1}, \cdots, t_{n}\right\} \subseteq T$; then $\left\{\phi\left(t_{1}\right), \cdots, \phi\left(t_{n}\right)\right\} \in$ $\mathcal{A}$ follows immediately from $\phi \in \mathcal{E}$.

We now use $(\mathrm{rTT})^{++}$to give a generalization of the Consistency Theorem as stated by Jech in [13]. In the original Consistency Theorem, the codomain of each function is $\{0,1\}$. To facilitate the proof, we will use the following lemma on constructing sets of finite character. 
Lemma 3.4 Let $T$ and $X$ be non-empty sets and let $\mathcal{E}$ be a collection of functions from finite subsets of $T$ into $X$ such that for all $\phi \in \mathcal{E}$ and all $F \subseteq$ $\operatorname{dom} \phi, \phi \mid F \in \mathcal{E}$. Let

$\mathcal{A}=\{\phi: \phi$ is a function from a subset of $T$ into $X$ and for all finite $F \subseteq$ $\operatorname{dom} \phi, \phi \mid F \in \mathcal{E}\}$.

Then

(1) $\mathcal{E} \subseteq \mathcal{A}$;

(2) if $\phi \in \mathcal{A}$ and dom $\phi$ is finite, then $\phi \in \mathcal{E}$;

(3) $\mathcal{A}$ has finite character.

Theorem 3.5 (Consistency, generalized) Let $\left\{X_{t}: t \in T\right\}$ be a collection of finite non-empty sets and let $\mathcal{E}$ be a collection of functions $\phi$ such that dom $\phi$ is a finite subset of $T$ and $\phi(t) \in X_{t}$ for all $t \in \operatorname{dom} \phi$ (each $\phi$ is a finite choice function). Assume that

(1) for each $F \in[T]^{<\omega}$, there exists $\phi \in \mathcal{E}$ such that dom $\phi=F$;

(2) if $\phi \in \mathcal{E}$ and $F \subseteq$ dom $\phi$, then $\phi \mid F \in \mathcal{E}$.

Then there is a choice function $\Phi$ for $\left\{X_{t}: t \in T\right\}$ such that for all $F \in[T]^{<\omega}$, $\Phi \mid F \in \mathcal{E}$.

Proof (using $(\mathrm{rTT})^{++}$). Let

$\mathcal{A}=\left\{\phi: \phi\right.$ is a function with dom $\phi \subseteq T, \phi(t) \in X_{t}$ for all $t \in \operatorname{dom} \phi$, and for all finite $F \subseteq \operatorname{dom} \phi, \phi \mid F \in \mathcal{E}\}$.

It suffices to find $\Phi \in \mathcal{A}$ with domain $T$. By Lemma $3.4, \mathcal{E} \subseteq \mathcal{A}$ and $\mathcal{A}$ has finite character (assumption (2) is used here). For each $t \in T$ let

$$
Z_{t}=\left\{\langle t, x\rangle: x \in X_{t}\right\} .
$$

To apply (rTT) $)^{++}$, it suffices to check $(++)$. Let $F=\left\{t_{1}, \cdots, t_{n}\right\} \subseteq T$. By (1), there is $\phi \in \mathcal{E}$ such that dom $\phi=F$. We now have

$$
\phi=\left\{\left\langle t_{1}, \phi\left(t_{1}\right)\right\rangle, \cdots,\left\langle t_{n}, \phi\left(t_{n}\right)\right\rangle\right\} \in \mathcal{A} \text { with }\left\langle t_{k}, \phi\left(t_{k}\right)\right\rangle \in Z_{t_{k}} \text { for } 1 \leq k \leq n .
$$

By $(\mathrm{rTT})^{++}$, there exists $\Phi \in \mathcal{A}$ such that $\Phi \cap Z_{t} \neq \varnothing$ for all $t \in T$; in other words, $\operatorname{dom} \Phi=T$ as required.

Rado's Selection Theorem (see [17]) is a corollary of the above generalized Consistency Theorem. 
Theorem 3.6 (Rado) Let $\left\{X_{t}: t \in T\right\}$ be a collection of finite non-empty sets. Assume that for each $B \in[T]^{<\omega}$, there is a choice function $\phi_{B}$ for $\left\{X_{t}: t \in B\right\}$ (the domain of $\phi_{B}$ is $B$ and $\phi(t) \in X_{t}$ for all $t \in B$ ). Then there is a choice function $\Phi$ for $\left\{X_{t}: t \in T\right\}$ such that for all $F \in[T]^{<\omega}$, there is a $B \in[T]^{<\omega}$ such that $F \subseteq B$ and $\Phi\left|F=\phi_{B}\right| F$.

Proof. Let $\mathcal{E}=\left\{\phi_{B} \mid F: F \subseteq B\right.$ and $\left.B \in[T]^{<\omega}\right\}$. The collection $\mathcal{E}$ satisfies (1) and (2) of the generalized Consistency Theorem. Let $\Phi$ be a choice function for $\left\{X_{t}: t \in T\right\}$ such that for all $F \in[T]^{<\omega}, \Phi \mid F \in \mathcal{E}$. In other words, $\Phi\left|F=\phi_{B}\right| F$, where $F \subseteq B$ and $B \in[T]^{<\omega}$ as required.

For the sake of completeness, we now sketch a proof that the Ultrafilter Theorem implies the axiom of choice for finite sets; the proof is just a simplified version of (UT) $\Rightarrow(\mathrm{CT})$ (see [13]).

Proof that $(\mathbf{U T}) \Rightarrow(\mathbf{A C F})$. Let $\left\{Z_{t}: t \in T\right\}$ be a collection of non-empty finite sets. We are required to prove the existence of a function $\Phi: T \rightarrow$ $\bigcup_{t \in T} Z_{t}$ such that $\Phi(t) \in Z_{t}$ for all $t \in T$. A partial choice function is a function $\phi: S \rightarrow \bigcup_{t \in T} Z_{t}$, where $S \subseteq T$ and $\phi(t) \in Z_{t}$ for all $t \in S$. Note that for every $F \in[T]^{<\omega}$, there is a partial choice function whose domain is $F$ (provable in $\mathrm{ZF}$ ). Let $\mathcal{E}$ be the collection of all partial choice functions. For each $F \in[T]^{<\omega}$ let

$$
\mathcal{E}_{F}=\{\phi: \phi \in \mathcal{E} \text { and } F \subseteq \operatorname{dom} \phi\} .
$$

Note that $\mathcal{E}_{F} \neq \varnothing$, and it is easy to check that for $F, G \in[T]^{<\omega}, \mathcal{E}_{F \cup G} \subseteq$ $\mathcal{E}_{F} \cap \mathcal{E}_{G}$. From this it follows that the collection $\left\{\mathcal{E}_{F}: F \in[T]^{<\omega}\right\}$ has the finite intersection property. By (UT), there exists $\mathcal{U} \subseteq P(\mathcal{E})$ such that

(1) $\mathcal{E}_{F} \in \mathcal{U}$ for all $F \in[T]^{<\omega}$;

(2) $\mathcal{U}$ has the finite intersection property;

(3) if $\mathcal{E}_{1} \cup \cdots \cup \mathcal{E}_{n} \in \mathcal{U}$, then $\mathcal{E}_{k} \in \mathcal{U}$ for some $k \leq n$.

Let $t \in T$ and let $Z_{t}=\left\{z_{1}, \cdots, z_{n}\right\}$. Then $\mathcal{E}_{\{t\}}=\mathcal{E}_{1} \cup \cdots \cup \mathcal{E}_{n}$, where for $1 \leq k \leq n$,

$$
\mathcal{E}_{k}=\left\{\phi: \phi \in \mathcal{E}_{\{t\}} \text { and } \phi(t)=z_{k}\right\} \text {. }
$$

By (3), there is a unique integer $k(t), 1 \leq k(t) \leq n$, such that $\mathcal{E}_{k(t)} \in \mathcal{U}$. Finally, define $\Phi: T \rightarrow \bigcup_{t \in T} Z_{t}$ by $\Phi(t)=z_{k(t)}$. 


\section{Theorems of logic from (rTT)}

We begin with a summary of basic ideas of propositional logic. Let FOR be the set of all formulas of propositional logic (use the connectives $\neg$ and $\vee)$. A truth assignment is a function $\phi: F O R \rightarrow\{T, F\}$ such that for all formulas $A$ and $B$ :

(1) $\phi(A) \neq \phi(\neg A)$;

(2) $\phi(A \vee B)=T$ if and only if $\phi(A)=T$ or $\phi(B)=T$.

Now let $\Gamma \subseteq F O R$.

- $\Gamma$ is satisfiable if there is at least one truth assignment $\phi$ such that $\phi(A)=T$ for every $A \in \Gamma$.

- $\Gamma$ is finitely satisfiable if every finite subset of $\Gamma$ is satisfiable.

- $\Gamma$ is consistent if there is no formula $A$ such that both $\Gamma \vdash A$ and $\Gamma \vdash \neg A$.

With this terminology, the Compactness Theorem states that every finitely satisfiable set is satisfiable, and the Model Existence Theorem states that every consistent set is satisfiable. Both finite satisfiability and consistency satisfy all of the hypotheses of (rTT). In other words:

(FS1) $\Gamma$ is finitely satisfiable if and only if every finite subset of $\Gamma$ is finitely satisfiable;

(FS2) if $\Gamma$ is finitely satisfiable and $A$ is any formula, then $\Gamma \cup\{A\}$ or $\Gamma \cup\{\neg A\}$ is finitely satisfiable;

(C1) $\Gamma$ is consistent if and only if every finite subset of $\Gamma$ is consistent;

(C2) if $\Gamma$ is consistent and $A$ is any formula, then $\Gamma \cup\{A\}$ or $\Gamma \cup\{\neg A\}$ is consistent.

The proof of $(\mathrm{C} 2)$ is perhaps the most difficult to verify and usually requires the Deduction Theorem; the precise details depend on the choice of the axioms and the rules of inference. Instead, let us verify (FS2).

Proof of (FS2). Let $\Gamma$ be finitely satisfiable, but suppose that neither $\Gamma \cup$ $\{A\}$ nor $\Gamma \cup\{\neg A\}$ is finitely satisfiable. Then there exist formulas $A_{1}, \cdots, A_{n}$, $B_{1}, \cdots, B_{k}$ in $\Gamma$ such that

(1) $\left\{A_{1}, \cdots, A_{n}, A\right\}$ is not satisfiable;

(2) $\left\{B_{1}, \cdots, B_{k}, \neg A\right\}$ is not satisfiable.

On the other hand, there is a truth assignment $\phi$ that satisfies

$$
\left\{A_{1}, \cdots, A_{n}, B_{1}, \cdots, B_{k}\right\}
$$


clearly $\phi$ satisfies $\left\{A_{1}, \cdots, A_{n}, A\right\}$ or $\left\{B_{1}, \cdots, B_{k}, \neg A\right\}$, a contradiction of (1) or (2).

We now prove the Model Existence Theorem.

Theorem 4.1 (Model Existence) If $\Gamma$ is consistent, then $\Gamma$ is satisfiable.

Proof. By (rTT), there is a set $\Delta$ of formulas such that

(1) $\Gamma \subseteq \Delta$;

(2) $\Delta$ is consistent;

(3) for every formula $A$, either $A \in \Delta$ or $\neg A \in \Delta$.

Define $\phi: F O R \rightarrow\{T, F\}$ by $\phi(A)=T \Leftrightarrow A \in \Delta$. Clearly $\phi$ satisfies $\Gamma$, and it is straightforward to check these two properties: $\phi(A) \neq \phi(\neg A)$; $\phi(A \vee B)=T$ if and only if $\phi(A)=T$ or $\phi(B)=T$.

It is a consequence of the Soundness Theorem (if $\Gamma \vdash A$, then $\Gamma \models A$ ) that finite satisfiability implies consistency; hence the Compactness Theorem follows from the Model Existence Theorem (in ZF). Alternatively, both theorems can be obtained from the following general theorem about properties of formulas.

Theorem $4.2(\mathrm{ZF}+\mathbf{r T T})$ Let $Q$ be a property of formulas of propositional logic such that

$(Q 1) Q$ has finite character $[\Gamma$ has property $Q$ if and only if every finite subset of $\Gamma$ has property $Q]$;

(Q2) $Q$ has the extension property with respect to $\neg[$ if $\Gamma$ has property $Q$ and $A$ is any formula, then $\Gamma \cup\{A\}$ or $\Gamma \cup\{\neg A\}$ has property $Q]$;

(Q3) for any formula $A$, the set $\{A, \neg A\}$ does NOT have property $Q$;

(Q4) for any formulas $A$ and $B$, the following sets do NOT have property $Q$ : $\{A \vee B, \neg A, \neg B\},\{A, \neg(A \vee B)\},\{B, \neg(A \vee B)\}$.

Then every set of formulas that has property $Q$ is satisfiable.

Proof. Similar to the proof of the Model Existence Theorem. Use (Q1) and (Q3) to prove $\phi(A) \neq \phi(\neg A)$; use (Q1) and (Q4) to prove $\phi(A \vee B)=T$ if and only if $\phi(A)=T$ or $\phi(B)=T$.

It is clear from the proof of the Model Existence Theorem that Lindenbaum's Theorem is a consequence of (rTT). In other words, we have:

Theorem 4.3 (Lindenbaum) Let $\Gamma$ be a consistent set of formulas. Then $\Gamma \subseteq \Delta$, where $\Delta$ is consistent and for every formula $A$, either $A \in \Delta$ or $\neg A \in \Delta$. 
We observe from the proof of the Model Existence Theorem that Lindenbaum's Theorem actually implies the Model Existence Theorem (in ZF). Finally, it is well known that the Compactness Theorem implies (BPI); see, for example [13]. So we can summarize the situation with respect to propositional logic as follows.

Theorem 4.4 (ZF) The following are equivalent.

(1) Boolean Prime Ideal Theorem;

(2) restricted Tukey-Teichmüller Theorem;

(3) Lindenbaum's Theorem;

(4) Model Existence Theorem;

(5) Compactness Theorem.

We briefly comment on the situation with respect to first-order logic. Let $L$ be a first-order language and let $S E N T$ be the set of all sentences of $L$. Let $\Gamma \subseteq S E N T$ be consistent, and suppose that we want to prove that $\Gamma$ has a model. By a well-known construction due to Henkin, we may assume that the language $L$ and the set $\Gamma$ satisfy the following: if $A$ is any formula of $L$ with exactly one free variable $x$, then there is a constant symbol $c$ of $L$ such that the sentence $\exists x A \rightarrow A_{x}[c]$ is in $\Gamma$. For details, see p. 46 of [21].

Note that the following holds in first-order logic: if $\Gamma$ is consistent and $A$ is a sentence, then $\Gamma \cup\{A\}$ or $\Gamma \cup\{\neg A\}$ is consistent. This means that (rTT) applies and we have Lindenbaum's Theorem: there exists $\triangle \subseteq S E N T$ such that $\Gamma \subseteq \Delta, \Delta$ is consistent, and for every sentence $A$ of $L, A \in \Delta$ or $\neg A \in \Delta$. Since $\Delta$ is also a Henkin theory, we can now assert that the canonical structure is a model of $\Delta$. Again see [21] for details.

In summary, we can add Lindenbaum's Theorem, the Model Existence Theorem, and the Compactness Theorem of first-order logic to the list of equivalent forms of the Boolean Prime Ideal Theorem.

Acknowledgement I am grateful to the referee for a very careful reading of this paper. Two suggestions are especially appreciated: the removal of a superfluous hypothesis in the original formulation of (rTT); the formulation of $(\mathrm{rTT})^{+}$as a generalization of (rTT). I would also like to thank Andreas Blass for many helpful suggestions.

\section{References}

[1] J. Alexander, Ordered sets, complexes, and the problem of bicompactifications, Proc. Nat. Acad. Sci. U.S.A. 22 (1939), 296-298.

[2] B. Banaschewski, The power of the ultrafilter theorem, J. London Math. Soc. 27 (1983), 193-202. 
[3] A. Blass, Prime ideals yield almost maximal ideals, Fund. Math. 127(1986), 57-66.

[4] R. Cowen, Some combinatorial theorems equivalent to the prime ideal theorem, Proc. Amer. Math. Soc. 41(1973), 268-273.

[5] R. Cowen, A Short Proof of Rado's Lemma, Journal of Combinatorial Theory (B) 12(1972), 299-300.

[6] E. Engeler, Eine Konstruktion von Modellerweiterungen, Z. Math. Logik Grundlagen Math. 5(1959), 126-131.

[7] R. Engelking, General Topology, Heldermann Verlag, Berlin, 1989.

[8] S. Feferman, Some applications of the notion of forcing and generic sets, Fund. Math. 56(1965), 325-345.

[9] O. Frink, Jr., Representations of Boolean algebras, Bull. Amer. Math. Soc. 47(1941), 755-756.

[10] J. D. Halpern and A. Lévy, The Boolean prime ideal theorem does not imply the axiom of choice, Axiomatic Set Theory, Proceedings of Symposia in Pure Mathematics 13(part 1), 83-134, AMS, Providence, R.I. 1971.

[11] L. Henkin, Metamathematical theorems equivalent to the prime ideal theorem for Boolean algebras, Bull. Amer. Math. Soc. 60(1954), 387-388.

[12] P. Howard and J. Rubin, Consequences of the Axiom of Choice, Mathematical Surveys and Monographs 59, American Mathematical Society, 1998.

[13] T. Jech, The Axiom of Choice, North Holland, Amsterdam, 1973.

[14] J. Kelley, General Topology, D. Van Norstrand, Princeton, N.J., 1955.

[15] S. Koppelberg, Handbook of Boolean Algebras, vol. 1, edited by D. Monk and R. Bonnet, North Holland, Amsterdam, 1989.

[16] I. Parovičenko, Topological equivalents of the Tihonov theorem, Soviet Math. Dokl. 10(1969), 33-34.

[17] R. Rado, Axiomatic treatment of rank in infinite sets, Canad. J. Math. 1(1949), 337-343.

[18] Y. Rav, Variants of Rado's Selection Lemma and their Applications, Math. Nachr. 79(1977), 145-165. 
[19] H. Rubin and J. Rubin, Equivalents of the Axiom of Choice, II, NorthHolland, Amsterdam, 1985.

[20] E. Schechter, Handbook of Analysis and Its Foundations, Academic Press, New York, 1997.

[21] J. Shoenfield, Mathematical Logic, Addison-Wesley, Reading, Mass., 1967.

[22] M. Stone, The theory of representations for Boolean algebras, Trans. Amer. Math. Soc. 40(1936), 37-111.

[23] O. Teichmüller, Braucht der Algebraiker das Auswahlaxiom?, Deutsche Math. 4(1939), 567-577.

[24] J. Tukey, Convergence and uniformity in topology, Ann. of Math. Studies 2, Princeton University Press, 1940.

[25] S. Wolk, On Theorems of Tychonoff, Alexander, and R. Rado, Proc. Amer. Math. Soc. 18(1967), 113-115.

Mathematics Department

Duke University

Durham, N.C. 27708

hodel@math.duke.edu 